\title{
The Galactic bulge: a review
}

\author{
Dante Minniti and Manuela Zoccali ${ }^{1} \dagger$
}

${ }^{1}$ Dept. of Astronomy \& Astrophysics, P. Universidad Catolica, Casilla 306, Santiago 22, Chile email: dante, mzoccali@astro.puc.cl

\begin{abstract}
The Milky Way is the only galaxy for which we can resolve individual stars at all evolutionary phases, from the Galactic center to the outskirt. The last decade, thanks to the advent of near IR detectors and 8 meter class telescopes, has seen a great progress in the understanding of the Milky Way central region: the bulge. Here we review the most recent results regarding the bulge structure, age, kinematics and chemical composition. These results have profound implications for the formation and evolution of the Milky Way and of galaxies in general. This paper provides a summary on our current understanding of the Milky Way bulge, intended mainly for workers on other fields.
\end{abstract}

Keywords. Galaxy: bulge, Galaxy: abundances, Galaxy: evolution, Galaxy: formation, Galaxy: kinematics and dynamics, Galaxy: stellar content, Galaxy: structure, Galaxy: disk, Galaxy: halo, globular clusters: general

\section{Introduction: The Questions}

How did the Milky Way form? Decades ago, the Galactic formation scenarios focused on the disk and the halo populations, because these were the components that astronomers knew something about. For example, in the classic works of Eggen et al. (1962) or Searle \& Zinn (1978) the bulge was not mentioned. All-sky optical maps (Fig. 1) did not show a clear, separate component in the inner Milky Way.

Yet upon looking at the new DIRBE or 2MASS near-infrared maps of the whole sky, it is evident that the Milky Way is a spiral galaxy with a peanut-shape bulge. The simultaneous observation that bulge stars were mainly old made it clear that to answer this question the attention had to shift towards what seems to be the first massive component to be formed in the Milky Way. This is a Copernican-like revolution on Galactic scales, and it is happening now! We see our Galaxy as if it were an external galaxy for the first time, and are fortunate to have such new perspective, and also to be able to provide specific answers to the many questions regarding its formation.

Note that we have only one object to study: our Galaxy. This is a fundamental limitation because we have to get the answers right. The advantage in the case of our Galaxy, of course, is that we can study the subject in detail: in no other galaxies the fundamental problems of Astronomy can be surpassed. Basically, these fundamental problems are: (1) The distance problem: we can resolve the components of the Milky Way into stars, and study them in situ, obtaining 3-D positions and motions, and detailed chemical compositions of individual stars; and (2) The timescale problem: we see an instant snapshot, and must infer histories from that. In our galaxy we can separate and date the components, using the main-sequence turn-offs of different stellar populations and clusters.

In spite of a small community working on the Galactic bulge, a revolution has occurred in this field in the last decade, with great progress in comparison with other fields of Astronomy. For the first time, we have the answers to important questions such as:

\section{$\dagger$ FONDAP Center for Astrophysics 15010003.}



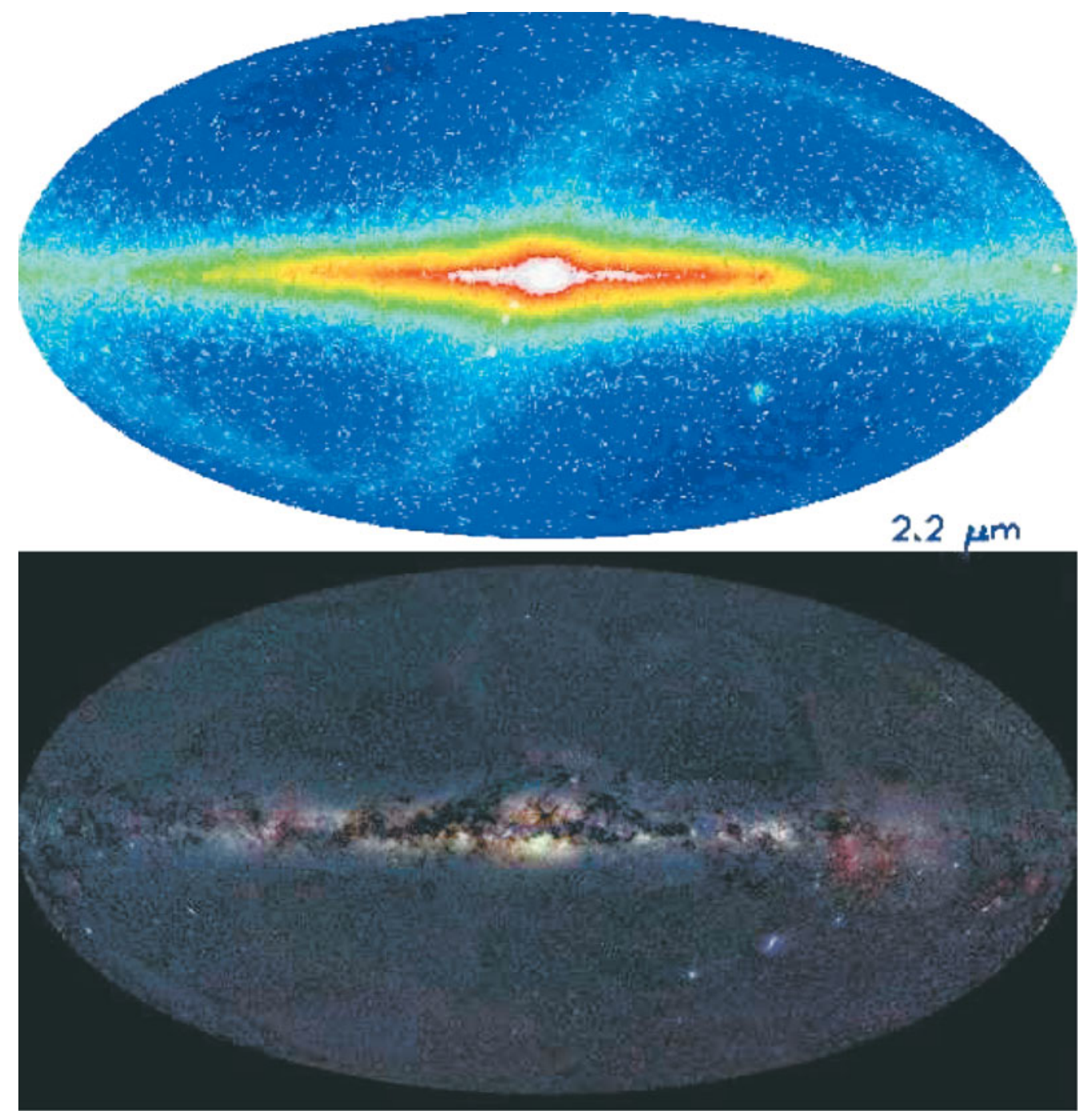

Figure 1. Top: Near-IR COBE-DIRBE map of the whole sky (Dwek et al. 1995). Bottom: Optical map for comparison (Copyright Axel Mallenhoff 2001). These sky maps illustrate why astronomers did not realize before the importance of the bulge.

1. Is the bulge a different Galactic component?

2. When did the Galactic bulge form?

3. How did it form?

4. Is there a radial gradient in the bulge?

5. Are there globular clusters associated with the bulge?

6. Are there planets in the bulge?

The $8 \mathrm{~m}$ class telescopes were built last decade, aiming to answer these questions. The proposed options were endless (bulge formation from secular evolution of the disk, from the halo, in a single burst, in several episodes, by slow accretion of smaller sub-units, etc). The evidence and the answers described below serve as a basis for understanding more distant galaxies which cannot be studied (dissected) in similar detail. 


\section{Structure of the Galactic Bulge}

The bulge is elongated, its barred structure was clearly shown by the DIRBE IR maps (Dwek et al. 1995), and by the clump giants (Stanek et al. 1994). This structure converges to a barred bulge of axes 1:0.35:0.26, inclined by 25 degrees with respect to the line of sight, with the nearest side located at positive longitudes (e.g. Rattenbury et al. 2007b).

Much of the progress on the bulge structure has come out as a byproduct of the microlensing experiments: from the microlensing events, from the color-magnitude diagrams (CMDs), and from the variable stars. These databases are treasures for the whole community to exploit.

While stellar populations trace light in galaxies, the microlensing optical depth is directly sensitive to the mass. The microlensing experiments have concentrated on bulge fields, discovering hundreds of events. The MACHO clump giants give a bulge optical depth $\tau=2.17 \times 10^{-6}$ at $l, b=1.5,-2.7$ (Popowski et al. 2005). The EROS and OGLE bulge results are in agreement (Hamadache et al. 2006, Sumi et al. 2006). The modeling of the inner barred mass distribution appears now in agreement (Gerhard 2005), with a bulge total mass of $1.6 \times 10^{10} M_{\odot}$.

However, there are still puzzles. Thousands of RR Lyrae have been found in the bulge. As classical tracers of old metal-poor populations, they may belong to the inner halo rather than to the bulge. At Baade's window the RR Lyrae have mean $[\mathrm{Fe} / \mathrm{H}]=-1.0$ (Walker \& Terndrup 1991). In fact, their distribution is not so clearly elongated as that of bulge clump giants, although a small barred structure may be present (Alcock et al. 1998, Collinge et al. 2006). Nevertheless, the strong central concentration of the RR Lyrae distribution (Fig. 2) illustrate the composite nature of the inner stellar population. An interesting possibility is that the metal-poor stars and globular clusters located in the inner bulge might be the oldest populations in the Galaxy (e.g. Mackey \& van den Bergh 2005).

There also appears to be a small bar (scale $\sim 600 \mathrm{pc}$ ) in the central region (Alard 2001, Niyishama et al. 2006), and a larger bar structure confined to the plane (e.g. Cole \& Weinberg 2002, Lopez-Corredoira et al. 2007). Indeed, much remains to be explored regarding the structure of the inner bulge. And more considering that the galaxy morphology might be a transient phenomenon (Steinmetz 2007), and that bars might appear and disappear (Combes 2007).

\section{Kinematics of the Galactic Bulge}

A great effort in the 90's was devoted to the measurement of the kinematics of bulge K-giants, representative of all bulge populations (as all giants go through this stage regardless of metallicity), using radial velocities. As shown in Fig. 3, the bulge is rotating, with a peak rotation of about $75 \mathrm{~km} / \mathrm{s}$ (Minniti et al. 1992, Harding \& Morrison 1993, Minniti 1996, Ibata et al. 1995, Beaulieu et al. 2006, Rich et al. 2007). It also has a large velocity dispersion (Terndrup et al. 1995, Minniti et al. 1996, Ibata et al. 1995), that decreases with Galactocentric distance.

The bulge kinematics are intermediate between a purely rotating system such as the Milky Way disk and a hot, non-rotating system like the Milky Way halo, that is supported by velocity dispersion. The so called $V_{\max } / \sigma$ diagram (Binney 1978) is a measure of how kinematically hot is a particular stellar system. This diagram has recently been adapted by Kormendy \& Kennicutt (2004) for external bulges. Fig. 4 shows an update of the bulge position in this diagram, with $\left(V_{\max } / \sigma\right)_{M W B}=0.67$ for the metal-rich bulge component, indicating that this system is kinematically hotter than the Milky Way disk 


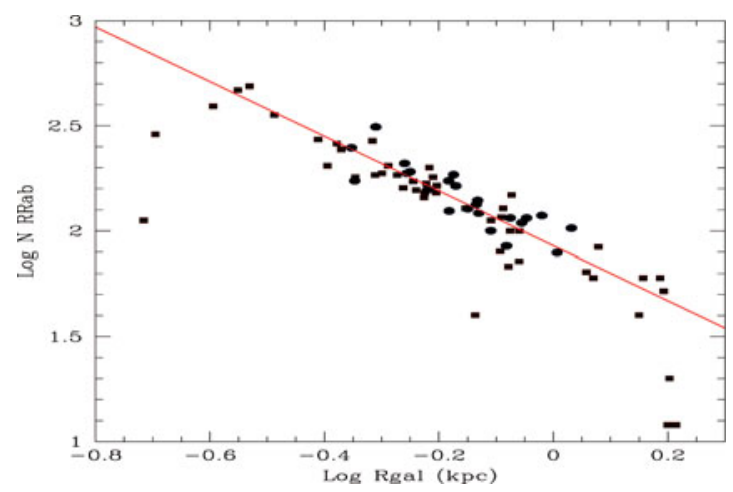

Figure 2. Number density of RR Lyrae type ab in the inner bulge vs Galactocentric distance from Alcock et al. (1998, circles) and Collinge et al. (2006, squares). The line is a spherical distribution with $\rho \propto r^{-2.3}$. The numbers per sqdeg have been normalized to Baade's window. Even though the innermost fields are still likely to be incomplete, the known RR Lyrae clearly show a very concentrated distribution. This figure shows that old and metal-poor populations are also present in the inner bulge regions.
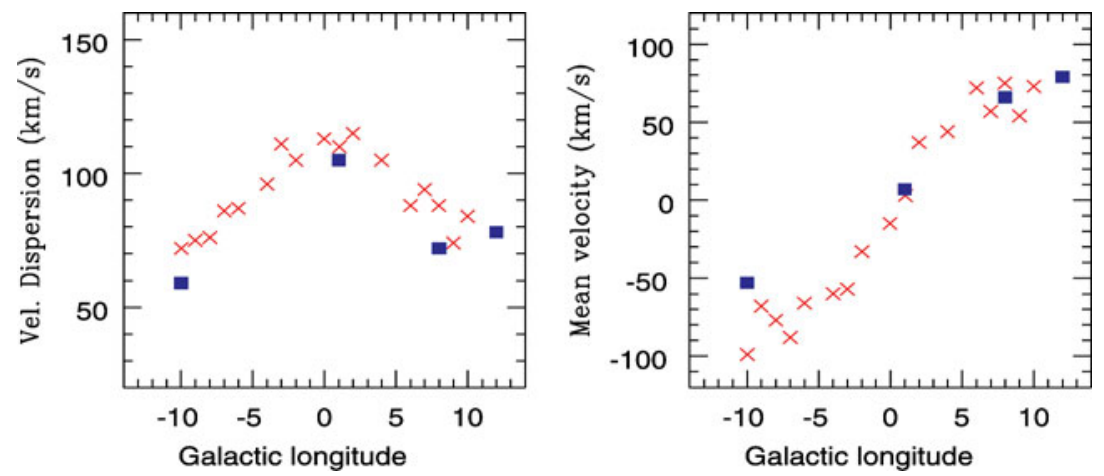

Figure 3. Bulge rotation from radial velocities of K-giants as measured by Minniti (1996), compared with the measurements of M-giants by Rich et al. (2007) (crosses). The latter have been corrected for the Solar motion around the Galaxy, which was not taken into account in the original paper.

(with $\left(V_{\max } / \sigma\right)_{M W D} \sim 2.2$ using the $\sigma_{M W D}$ measured by Morrison et al. 1990), but colder than the halo with $\left(V_{\max } / \sigma\right)_{M W H} \sim 0.15$ (Minniti 1996, Battaglia et al. 2005).

The proper motions were measured in Baade's window by Spaenhauer et al. (1992), confirming the high velocity dispersion of M-giants. More recent proper-motion measurements from the ground and space are following their steps (Kuijken \& Rich 2002, Soto et al. 2007, Vieira et al. 2007, Rattenbury et al. 2007a), and this is a field where a lot of progress is expected: we need orbits of bulge stars, to be able to distinguish between a possible inner disk/halo component and the true bulge.

\section{The Bulge Globular Cluster System}

The metal-rich globular clusters in the central regions of the Milky Way share the kinematics, spatial distribution, and composition of the bulge field stars (Minniti 1995, Barbuy et al. 1998, Côté 1999, Bica et al. 2006). This realization was very important because it allowed us to trace the bulge stellar population by means of its globular clusters. Typical bulge globular clusters like NGC6553, NGC6528, NGC6441, have been well 


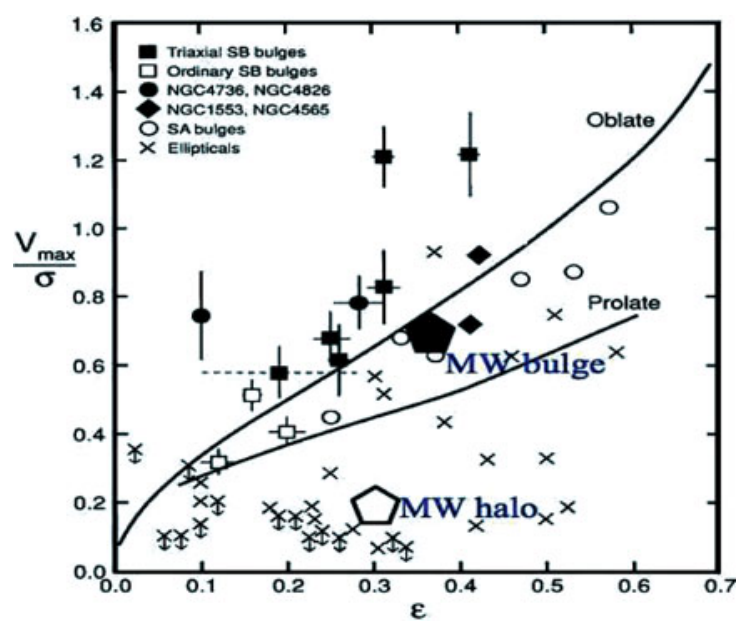

Figure 4. Milky Way bulge and halo position in the Binney (1978) diagram adapted from Minniti (1996) and Kormendy \& Kennicutt (2004).

studied, with accurate distances, reddenings, ages, individual star memberships and detailed chemical compositions (e.g. Barbuy et al. 2005, Zoccali et al. 2004, Alves-Brito et al. 2006), and serve now as standards for bulge studies. The detailed chemical compositions of the old metal-poor globular clusters present in the bulge are being measured as well (Barbuy et al. 2006, 2007).

\section{The Age of the Galactic Bulge}

The bulk of the metal-rich stellar population of the Galactic bulge is old, with an age of $t=10 \pm 2.5$ Gyr (Ortolani et al. 1995, Zoccali et al. 2003). This result has been obtained by direct comparison of bulge field stars with metal-rich globular clusters.

The deepest CMD of the bulge to date was obtained with the ACS at HST by Sahu et al. (2006). This diagram, shown in Fig. 5, is consistent with such an old age. These data were acquired during an HST large programme to find planetary transits in the bulge: the SWEEPS project (Sagittarius Window Eclipsing Extrasolar Planets Search). Sahu et al. (2006) found 16 transiting planetary size objects, two of which were confirmed to be real planets using radial velocities.

In spite of that, there are tracers of a younger population such as $\mathrm{OH} / \mathrm{IR}$ stars, bright AGB variables, etc., that appear to be mostly confined to the Galactic plane (e.g. van Loon et al. 2003). The bulk of the bulge, however, is old.

\section{The Chemical Composition of the Galactic Bulge}

In principle, the measurement of the age distribution of bulge stars should allow us to derive its star formation rate, and thus to answer at least the question on when (if not how) did the bulge form. In practice, the disk contamination right on top of the bulge turnoff, coupled with the metallicity, distance and reddening dispersions did not permit the dating of the bulge to better than \pm 2.5 Gyr. Complementary observation of the metallicity distribution and the detailed element ratios allowed to constrain the formation timescale, the star formation rate, initial mass function, the infall of primordial material, and the possible occurrence of accretion of smaller sub-units. 


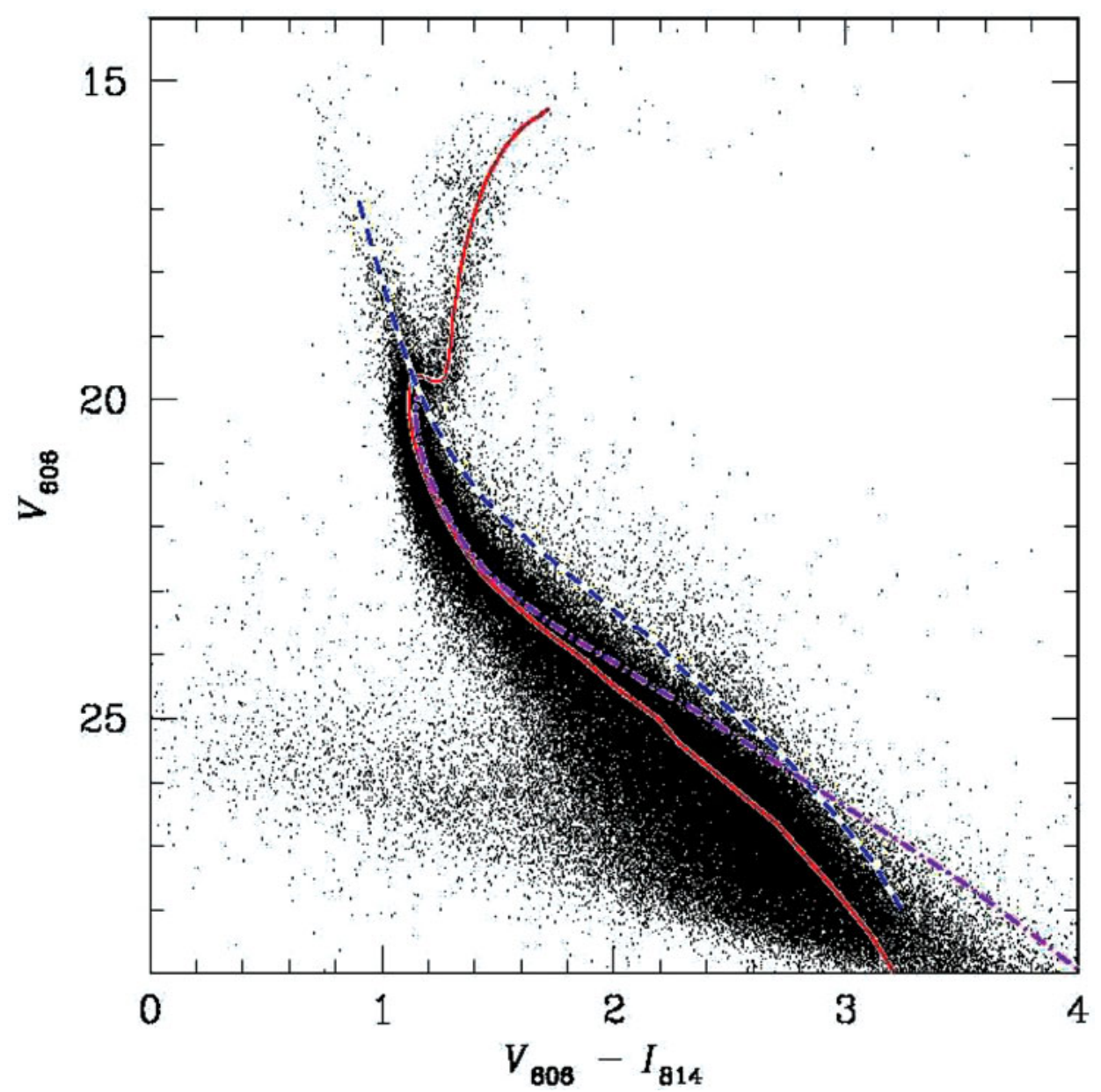

Figure 5. The deepest bulge CMD from 86,106 and 89,835 sec in the F606W (V) and F814W (I) bands, obtained with the ACS at the HST on a 202" " $\times 202$ " field at $l, b=1.25,-2.65$ (Sahu et al. 2006). About 245,000 stars are plotted down to $V=29$. A Solar metallicity isochrone of 10 Gyr is plotted with the red line, fitting the bulk of the bulge population. The foreground disk is fit with an unevolved main sequence shown by the blue dashed line. A super metal-rich isochrone with $[\mathrm{Fe} / \mathrm{H}]=+0.5$ is also shown with the magenta dot-dashed line.

\subsection{The Metallicity of the Galactic Bulge}

The bulge metallicity is far from that of a simple stellar population like a globular cluster, and therefore a large number of stars need to be measured in order to sample the whole metallicity range. Being high resolution spectroscopy of a large number of stars prohibitive, in the past people would determine the metallicity distribution via low resolution spectra for a few hundred stars (e.g. Sadler et al. 1996, Ramirez et al. 2000) and sometimes obtaining high resolution spectra only for a few dozen stars, used as calibrators (McWilliam \& Rich 1994, Fulbright, McWilliam \& Rich 2006). They would all agree that the Galactic bulge was metal rich, but there was contradictory evidence regarding the mean, the spread and the shape of the distribution.

Zoccali et al. (2007) and Lecureur et al. (2007b) have now obtained for the first time the bulge metallicity distribution from a sample of $\sim 1000 \mathrm{~K}$-giants in 4 bulge windows, all observed with high dispersion spectra $(R>20000)$, with FLAMES at VLT. The 


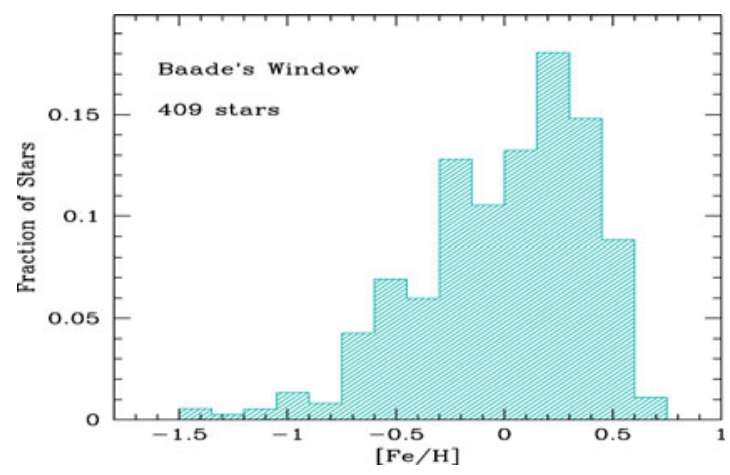

Figure 6. Metallicity distribution of 409 RGB and clump K-giants in the bulge from Zoccali et al. (2007) and Lecureur et al. (2007b), all measured for the first time with high-resolution spectra. This is in a firm metallicity scale as they observed simultaneously a dozen stars of known globular clusters located in the bulge.

resulting metallicity distribution for Baade's Window is shown in Fig. 6. Its shape is well reproduced by the most recent chemical evolution models (Ballero et al. 2006) assuming a very high star formation efficiency, short infall timescale, and a rather top-heavy initial mass function (as measured in the bulge by Zoccali et al. 2000, for $1.0<M / M_{\odot}<0.15$ ).

\subsection{The Detailed Element Abundances of the Galactic Bulge}

Different teams were pursuing the detailed element abundances of bulge giants using high dispersion spectrographs, following the pioneering effort by McWilliam \& Rich (1994). Recently, Rich \& Origlia (2005) found that $\alpha$-elements are enhanced to $[\mathrm{O} / \mathrm{Fe}]=+0.4$ for 13 giants within a narrow metallicity range around $[\mathrm{Fe} / \mathrm{H}]=-0.2$. Later on, Zoccali et al. (2006) and Lecureur et al. (2007a) measured oxygen, magnesium, sodium and aluminum in a sample of $50 \mathrm{~K}$-giants with $[\mathrm{Fe} / \mathrm{H}]$ covering a wide metallicity range, from -0.8 to +0.3 using the UVES spectrograph at the VLT. The result is shown in Fig. 7, where it is evident that bulge stars have larger $[\mathrm{O} / \mathrm{Fe}]$ and $[\mathrm{Mg} / \mathrm{Fe}]$ than both thin and thick disk stars. This is the signature of a chemical enrichment by massive stars, progenitors of SNII, with little or no contribution from SNIa, and thus of a shorter formation timescale of the bulge with respect of both disk components. In this sense, the bulge (including its globular clusters) is the most extreme Galactic population.

These results agree with the predictions of recent bulge formation models all assuming a fast formation at early epochs (Ballero et al. 2006, Immeli et al. 2004). Most importantly, they were confirmed by independent teams: Fulbright et al. (2007) analyzed $27 \mathrm{~K}$ giants, and Cunha \& Smith (2007) observed 7 giants, all with enhanced Oxygen with respect to the other Galactic components, at all metallicities.

\section{The Stellar Population Gradient of the Galactic Bulge}

Is there a stellar population gradient in the bulge? The CMDs of the microlensing surveys that mapped a large fraction of the bulge showed significant differences with increasing distance from the center and from the plane. The most conspicuous effect is that the red giant branch gets bluer and narrower moving away from the Galactic center (Fig. 7). This observed gradient can be due to: the different contributions of the bulge vs disk vs halo, reddening/extinction inhomogeneities, crowding, a variation in chemical composition, age differences, or a combination of any of the above. 

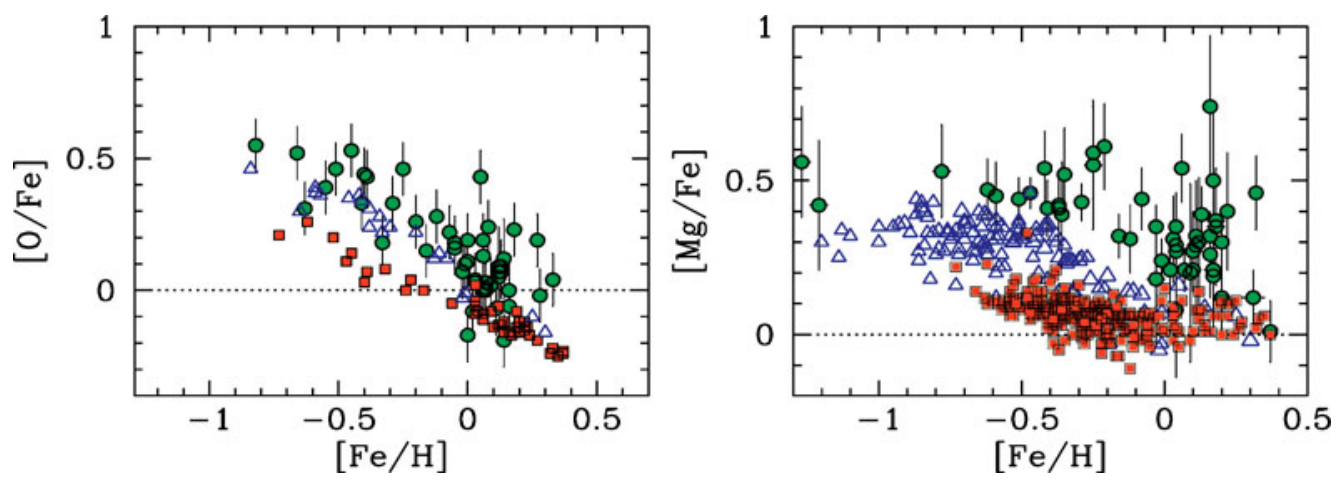

Figure 7. Oxygen and magnesium over iron ratios as measured from high dispersion spectra of bulge K giants (Zoccali et al. 2007, Lecureur et al. 2007a). Green circles with error bars are bulge stars, compared with thick (blue triangles) and thin (red squares) disk stars. These results clearly indicate that the bulge formed as a separate component by a rapid chemical enrichment.

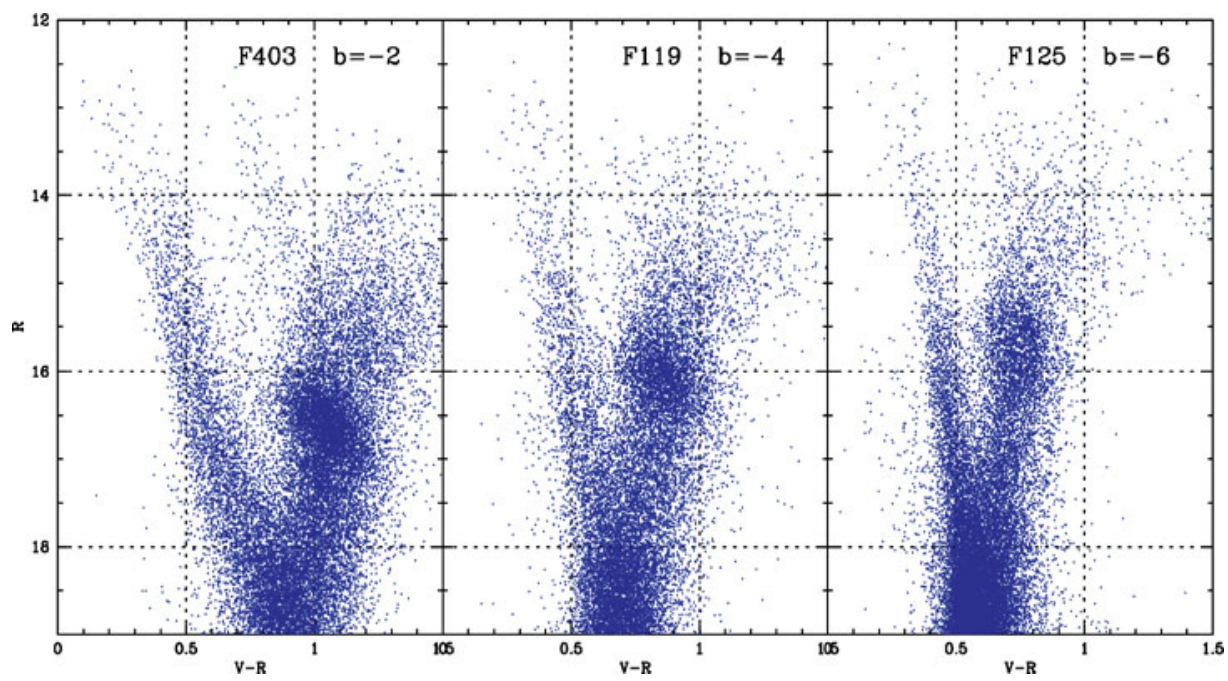

Figure 8. CMDs for three MACHO fields along the bulge minor axis. The change in the stellar population is evident, with the RGB getting bluer and narrower away from the Galactic center. This cannot only be due to reddening, and is explained by the metallicity gradient measured using high dispersion spectroscopy of K giants (Zoccali et al. 2007, Lecureur et al. 2007b).

Several pieces of evidence pointed towards a metallicity gradient (Minniti et al. 1995), however a very large spread existed between different authors' measurements on the same fields. Furthermore, recent analysis (Ramirez et al. 2000; Rich, Origlia \& Valenti 2007) suggested the absence of a gradient in the inner region $(b=1-4$ degrees, i.e., inside Baade's Window). The final answer comes from the homogeneous iron abundances from high dispersion spectroscopy of nearly $1000 \mathrm{~K}$-giants (Zoccali et al. 2007 and Lecureur et al. 2007b). The bulge has a metallicity gradient, with the metallicity decreasing by 0.25 dex along the Galactic minor axis between $b=-4$ and $b=-12 \mathrm{deg}$.

\section{Conclusions: The Answers}

For the first time, we have the answers to the following basic questions: 
1. Is the bulge a different component? Yes, based on all the evidence available, the bulge is a distinct Galactic component, with different kinematics and compositions from the thin disk, the thick disk and the halo.

2. How did the Galactic bulge form? It formed on a short timescale ( $1 \mathrm{Gyr})$, as demonstrated by the $\alpha$-element enrichment. Despite the presence of the bar, models like a bulge formation via secular evolution of the disk can be firmly excluded.

3. What is the age of the bulge? The bulk of the stellar population is $\sim 10$ Gyr old. However, there are traces of a small fractions of intermediate-age stars, and of metal-poor stars. The latter might well be the oldest population in the Galaxy.

4. Is there a gradient in the bulge? Yes, there is a stellar population gradient as shown for example by the CMDs. Now it is found that this gradient is mostly due to metallicity, which decreases along the Galactic minor axis.

5. Are there globular clusters associated with the bulge? Yes, there is a population of metal-rich globular clusters in the central regions that share the kinematics, spatial distribution, and composition of the bulge field stars.

6. Are there planets in the bulge? Even though this question seems to belong to another field, another of the recent advances was the discovery of planets in the bulge by HST. The available data suggests that giant planets are as numerous in the bulge as they are in the Solar neighborhood.

These revolutionary advances that impact the whole of extragalactic Astronomy cannot be attributed to the success of a single group, but to the combined contributions of different teams. Where controversy was present before, today similar answers are given. Progress has occurred!

\section{Future Dreams}

We are now dreaming of the bulge science that would be enabled with the next generations of $4-8 \mathrm{~m}$ class survey telescopes, and of 20-40 m class ELTs. Our dreams are that the survey telescopes like VISTA will allow us to map the whole bulge, unveiling its entire structure and stellar populations (the VVV survey), and that the ELTs will allow us to measure the chemical compositions of bulge turn-off stars as function of their ages.

\section{References}

Alard, C. 2001, A\&A, 379, L44

Alcock, C. et al. 1998, ApJ, 492, 190

Alves-Brito, A., et al. 2006, A\&A, 460, 269

Ballero, S. K., Matteucci, F., \& Ciappini, C. 2006, NewA, 11, 306

Barbuy, B., et al. 2005, Highlights in Astronomy, Vol. 13, p. 159

Barbuy, B., et al. 2006, A\&A, 449, 349

Barbuy, B., et al. 2007, AJ, 134, 1613

Barbuy, B., Bica, E., \& Ortolani, S. 1998, A\&A, 333, 117

Battaglia, G., et al. 2005, MNRAS, 370, 1055

Beaulieu, S. F., et al. 2000, AJ, 120, 855

Bica, E., et al. 2006, A\&A, 450, 105

Binney, J. 1978, MNRAS, 183, 501

Cole, A. A. \& Weinberg, M. D. 2002, ApJ, 574, L43

Collinge, M. J., Sumi, T., \& Fabrycky, D. 2006, ApJ, 651, 197

Combes, M. 2007, in IAU Conf. 245, in press

Côté, P. 1999, AJ, 188, 406

Cunha, K. \& Smith, V. V. 2006, ApJ, 651, 491 
Dwek, E., et al. 1995, ApJ, 604, L93

Eggen, O., Lynden-Bell, D., \& Sandage, A. R. 1962, ApJ, 136, 748

Fulbright, J. P., McWilliam A., \& Rich R. M., 2006, ApJ, 636, 821

Gerhard, O. 2006, EAS Publ. Series Vol. 20, (EDP Sciences), p. 89

Hamadache, C., et al. 2006, A\&A, 545, 185

Harding, P. \& Morrison, H. 1993, in IAU Symp. 153 "Galactic Bulges", eds. H. Dejonghe \& H. Habing (Dordrecht: Kluwer), 57

Ibata, R. \& Gilmore, G. 1995, MNRAS, 275, 591

Immeli, A., Samland, M., Gerhard, O. \& Westera, P. 2004, A\&A 413, 547

Kormendy, J. \& Kennicutt, R. C. 2004, ARA\&A, 42, 603

Kuijken, K. \& Rich, M. 2002, ApJ, 124, 2054

Lecureur, A., et al. 2007a, A\&A, 465, 799

Lecureur, A., et al. 2007b, A\&A, to be submitted

Lopez-Corredoira, M. et al. 2007, AJ, 133, 154

McWilliam, A. \& Rich, R. M. 1994, ApJ, 91, 749

Minniti, D. 1996, ApJ, 459, 175

Minniti, D., et al. 1992, ApJ, 393, L47

Minniti, D., et al. 1995, MNRAS, 277, 1293

Morrison, H., Flynn, C., \& Freeman, K. C. 1990, AJ, 100, 119

Ortolani, S., et al. 1995, Nature, 377, 701

Popowski, P., et al. 2005, ApJ, 631, 879

Ramirez, S. V. et al. 2000, AJ, 120, 833

Rattenbury, N. J., et al. 2007a, MNRAS, 378, 1165

Rattenbury, N. J., et al. 2007b, MNRAS, 378, 1064

Rich, M. 1990, ApJ, 362, 604

Rich, M. \& Origlia, L. 2005, ApJ, 634, 1293

Rich, M., et al. 2007, ApJ, 658, L29

Sadler, E., Rich, M., \& Terndrup, D. 1996, AJ, 112, 171

Sahu, K., et al. 2006, Nature, 443, 534

Searle, L. \& Zinn, R., 1978, ApJ, 225, 357

Soto, M. et al. 2007, ApJ, 665, L31

Spaenhauer, A., Jones, B. F., \& Whitford, A. E. 1992, AJ, 103, 297

Stanek, K. Z., et al. 1994, ApJ, 429, L73

Steinmetz, M. 2007, in IAU Conf. 245, in press

Sumi, T., 2006, ApJ, 636, 240

Terndrup, D., Sadler, E., \& Rich, M. 1995, AJ, 110, 1774

Mackey, A. D. \& van den Bergh, S. 2005, MNRAS, 360, 631

van Loon, J. Th., et al. 2003, MNRAS, 338, 857

Vieira, K., et al. 2007, ApJ, in press

Walker, A. \& Terndrup, D. M. 1991, ApJ, 378, 119

Zhao, H.-S. 1996, MNRAS, 283, 149

Zoccali, M., et al. 2000, ApJ, 530, 418

Zoccali, M., et al. 2003, A\&A, 399, 931

Zoccali, M., et al. 2004, A\&A, 423, 507

Zoccali, M., et al. 2006, A\&A, 457, L1

Zoccali, M. et al. 2007, A\&A, submitted 